\title{
Primary Retroperitoneal Filariasis Presenting with Acute Abdomen
}

\author{
Maram Z. Nached Labib S. Al-Ozaibi \\ General Surgery Department, Rashid Hospital, Dubai Health Authority, Dubai, United Arab Emirates
}

\section{Keywords}

Acute abdomen - Filarial dance sign · Filariasis - Lymphatic filariasis - Primary retroperitoneal filariasis

\begin{abstract}
Lymphatic filariasis is a common tropical parasitic infection caused by the Nematoda Filarioidae family. Filariasis predominantly affects the lymphoreticular system, leading to lymphedema, elephantiasis, hydrocele, and chyluria. However, its presentation as retroperitoneal cyst is very rare with a reported incidence rate of $1 / 105,000$. We present a rare case of a previously healthy 26-year-old male presenting with abdominal and groin pain associated with chills and vomiting. Computerized tomography scan showed extensive cystic retroperitoneal lesion and ultrasound demonstrated "Dancing Filarial Sign." Retroperitoneal filariasis can present without peripheral filarial signs. Hence, it should be considered in the differential diagnosis of acute abdominal pain of a patient who is originally from an endemic area.
\end{abstract}

(C) 2021 The Author(s)

Published by S. Karger AG, Basel

karger@karger.com www.karger.com/dmj

Karger"

GOPEN ACCESS
C) 2021 The Author(s)

Published by S. Karger AG, Basel

This article is licensed under the Creative Commons Attribution NonCommercial-NoDerivatives 4.0 International License (CC BYNC-ND) (http://www.karger.com/Services/OpenAccessLicense) Usage and distribution for commercial purposes as well as any distribution of modified material requires written permission.

\section{Introduction}

Lymphatic filariasis is a common tropical parasitic infection caused by nematodes: Wuchereria bancrofti, Brugia malayi, and Brugia timori, transmitted by a Vector (mosquitoes), affecting 120 million people around the world [1]. After transmission the larvae then reside in the lymphatics and lymph nodes mature into adult worms and release microfilariae into the circulation [2].

In chronic stages, Filariasis commonly presents as lymphedema, elephantiasis and hydrocele. However, its presentation as retroperitoneal cyst is very rare with a reported incidence rate of $1 / 105,000$ [3]. In this report, we present a rare case of primary retroperitoneal filariasis presenting with features of acute abdomen.

\section{Case Presentation}

A 26-year-old previously healthy male presented to the Emergency Department with a 2-day history of periumbilical abdominal pain. The pain started in the left groin area and then shifted to the periumbilical region, radiating to the back and associated with chills and vomiting, no history of anorexia, fever, or rash. Along with this the patient denied any history of vigor- 


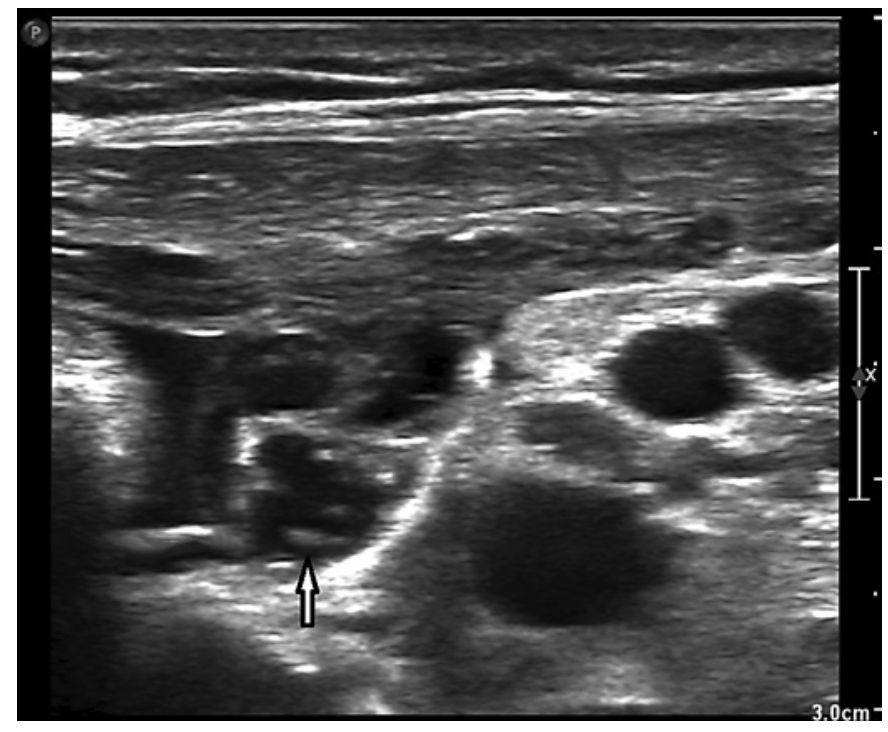

Fig. 1. Ultrasound image. The arrow points to the larva in the cyst.

ous exercise or minor trauma to the testes. This is the first attack of such pain.

On examination lower abdominal tenderness was noted, with no rebound tenderness, or renal angle tenderness. To rule out testicular origin of pain, scrotal examination was done, which only demonstrated boggy and thick spermatic cord. Rest of the systemic examination was within normal limits.

In the workup of patients with acute abdominal pain several differentials must be considered, and they can be ruled out by correlating the clinical picture, examination findings and appropriate investigations. In this case, our differentials initially included acute appendicitis, pancreatitis, ureteric colic, and testicular torsion.

Investigations revealed a white blood cell (WBC) count of $16.4 \times 10^{3} / \mu \mathrm{L}$ with left shift neutrophils $93.0 \%$, and normal eosinophilic count (1\%). C-reactive protein was raised $196.0 \mathrm{mg} / \mathrm{L}$ (reference range $<5.0 \mathrm{mg} / \mathrm{L}$ ) and procalcitonin $33.64 \mathrm{ng} / \mathrm{mL}$ (reference range $<0.05 \mathrm{ng} / \mathrm{mL}$ ). Amylase and lipase were within normal range. Kidney function tests were within normal limits along with normal Urinalysis. COVID-19 test was done in the view of patients presentation during the COVID pandemic and his elevated inflammatory markers which was negative.

Besides this, an abdominal ultrasonography showed multiple cystic structures with multiple curvilinear echogenic undulating structures with Filarial dance sign (see online suppl. Video 1; see www.karger.com/doi/10.1159/000514510 for all online suppl. material; Fig. 1). A Contrast Enhanced Computerized Tomography was done to know the extent of the lesion and showed cystic density lesion in the retroperitoneum in para-aortic area encasing the aorta and inferior vena cava up to level of renal vein and down to para iliac vessels and into the inguino-scrotal region (Fig. 2, 3). The patient was started on Albendazole $400 \mathrm{mg}$ oral twice per day for 7 days and Rifampicin $600 \mathrm{mg}$ oral once daily for 7 days. The patient responded well, became asymptomatic and discharged in a good condition.

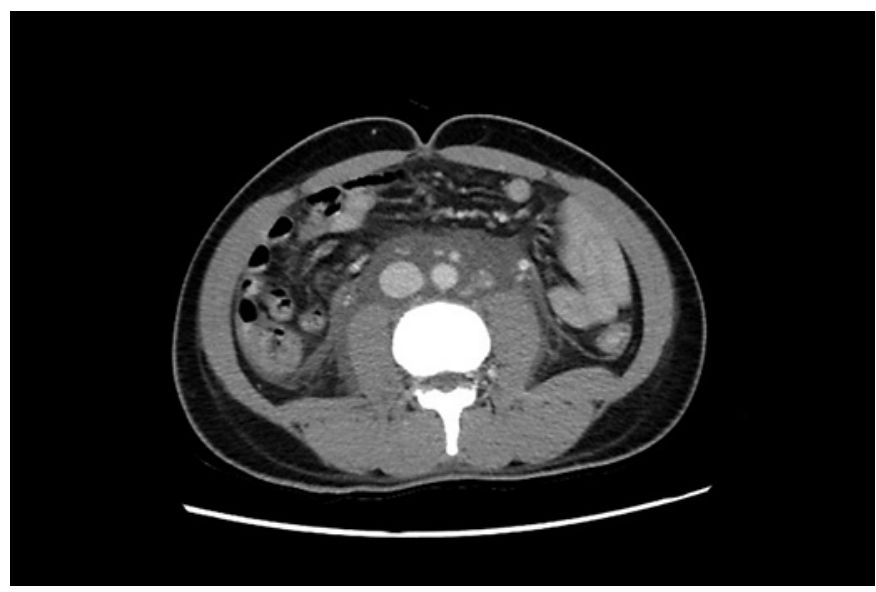

Fig. 2. Retroperitoneal cystic density lesion encasing the vessels.

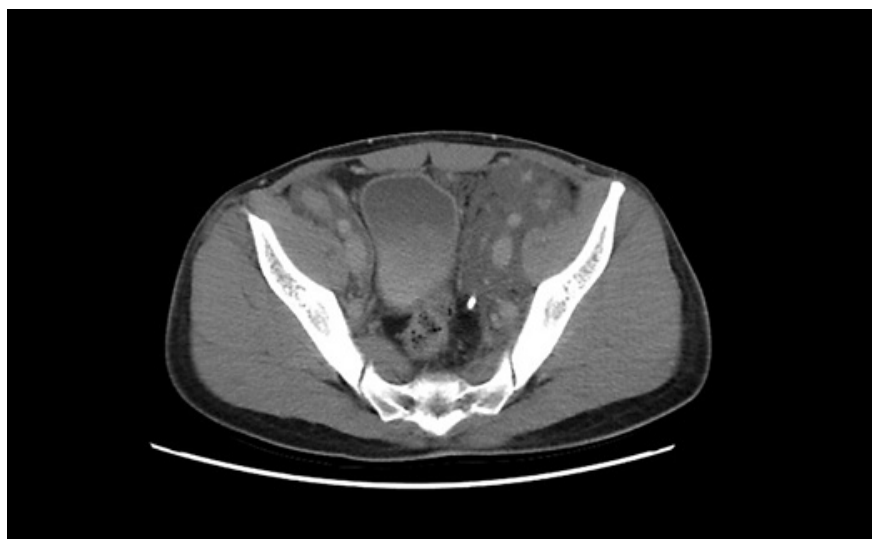

Fig. 3. Retroperitoneal infiltrating lesion encasing the iliac vessels.

\section{Discussion}

Lymphatic filarial disease is the second leading parasitic cause of disability and manifests in a wide variety of severe clinical pathologies. Filariasis targets specifically the lymphatic vessels and impairs the lymph flow through dilation and loss of contractile strength of the vessels leading to valvular dysfunction and eventually lymphedema. The damage is further advanced by secondary bacterial infection causing dermato-lymphangio-adenitis. Frequent attacks of lymphangio-adenitis cause lymphedema that later evolves into elephantiasis and hydrocele $[1,2]$.

Filarial infection remains subclinical in two-thirds of the cases, and the minority go on to develop overt disease [2]. The acute manifestations of filariasis are characterized by retrograde adenolymphangitis, and involvement of male urogenital system in the form of Funiculitis, epi- 
didymitis and orchitis. The chronic sequelae of filariasis include lymphedema, elephantiasis, hydrocele, chylous ascites, or chyluria in case of ruptured dilated retroperitoneal or urinary tract lymphatics [1].

Filariasis presenting as retroperitoneal mass is rare, even in areas where it is endemic. The pathogenesis of the lesion remains uncertain. Obstructed lymphatic vessels, rupture of lymphatics causing extravasation of chyle and the presence of ectopic lymphatic tissue have been suggested as possible aetiologies [3]. The written literature about retroperitoneal filariasis is limited to few case reports with clinical presentation as dull abdominal pain with or without accompanying symptoms such as hematuria or groin pain due to pressure effect $[4,5]$. Few reported cases of some rare presentations of filariasis includes acute abdominal pain due to retroperitoneal extension, large retroperitoneal cysts, female infertility due to tubo-ovarian involvement, male infertility and hydronephrosis [3-7]. The definitive diagnosis for filariasis remains through demonstration of microfilaria in peripheral blood smear, hydrocele fluid, or in other body fluids. However, due to diurnal variation, assays for circulating antigens of $W$. bancrofti or PCR for the parasitic DNA appear to be more convenient [8].

The treatment remains a course of antifilarial medications, but it is important to assess for other concomitant infections and provide the treatment accordingly. Diethylcarbamazine is a CDC approved drug for treatment of filariasis. It has shown to kill approximately $50 \%$ of adult worms that further decreases the microfilaria burden [9]. Other drugs such as Albendazole, Ivermectin and Doxycycline have shown activity against micro and macro-filaria [8]. In our case the patient was treated with a 7 days course of Albendazole and Rifampicin with which he improved.

In our case, a definitive diagnostic test was not performed. However, correlation between the presentation, labs and imaging studies was done to narrow differentials of acute abdominal pain, which in this case showed leukocytosis, elevated inflammatory markers, and imaging features of lymphangiectasia caused by filariasis along with the pathognomonic "dancing larvae sign."

\section{Conclusion}

Retroperitoneal filarial mass lesion presenting with acute abdominal pain, without peripheral filarial signs is rare even in endemic areas. Hence, it should be considered in the differential diagnosis of acute abdominal pain of a patient who is from an endemic area. Surgeons should be aware of this rare and medically treatable cause of acute abdomen to avoid the unnecessary surgical intervention.

\section{Statement of Ethics}

Written informed consent was obtained from the patient for publication of this case report and any accompanying images.

\section{Conflict of Interest Statement}

The authors have none to declare.

\section{Funding Sources}

The authors did not receive any funding.

\section{Author Contributions}

M.Z.N. wrote the manuscript, L.S.A. collected the data and reviewed the final report.

\section{References}

1 Nutman TB. Insights into the pathogenesis of disease in human lymphatic filariasis. Lymphat Res Biol. 2013;11(3):144-8.

2 Chakraborty S, Gurusamy M, Zawieja DC, Muthuchamy M. Lymphatic filariasis: perspectives on lymphatic remodeling and contractile dysfunction in filarial disease pathogenesis. Microcirculation. 2013;20(5):349-64.

3 Ganesan S, Galodha S, Saxena R. Retroperitoneal cyst: an uncommon presentation of filariasis. Case Rep Surg. 2015; 2015. Article ID 674252, 3 pages.
4 Kanthala LK, Reddy S, Ghuman SS, Kakar A, Nundy S. Acute filarial lymphangitis presenting with acute abdominal pain. J Case Rep. 2020: 29-32.

5 Metha NN, Dalavi VS, Mehta NP. Filariasis presenting as acute abdominal pain: the role of imaging and image-guided intervention in diagnosis. Radiol Case Rep. 2015;10(2):1125.

6 Chakravarthy S, Chakravarty R. Diagnosis of tubo-ovarian filariasis with retroperitoneal extension: a radiological challenge. J Family Med Prim Care. 2019;8(6):2144.
7 Connor MJ, Habib A, Wen D, Kubba F, Raza A. Filariasis an unusual cause of retroperitoneal fibrosis, hydronephrosis, and infertility in a young adult male. J Endourol Case Rep. 2019;5(3):1136.

8 Kasper D, Fauci A, Hauser S, Longo D, Jameson J, Loscalzo J. Harrison's principles of internal medicine. 20th ed. New York: McGraw-Hill Education/Medical; 2018. p. 1629-32.

9 Klion AD, Ottesen EA, Nutman TB. Effectiveness of diethylcarbamazine in treating loiasis acquired by expatriate visitors to endemic regions: long-term follow-up. J Infect Dis. 1994;169(3): 604-10. 